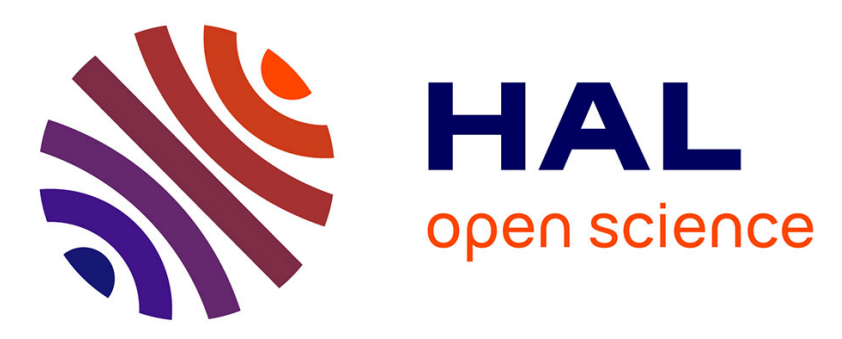

\title{
Identification of equivalent topography in an open channel flow using Lagrangian data assimilation
}

Marc Honnorat, Jerome Monnier, Nicolas Riviere, Etienne Huot, François-Xavier Le Dimet

\section{- To cite this version:}

Marc Honnorat, Jerome Monnier, Nicolas Riviere, Etienne Huot, François-Xavier Le Dimet. Identification of equivalent topography in an open channel flow using Lagrangian data assimilation. Computing and Visualization in Science, 2010, 13 (3), pp.111-119. 10.1007/s00791-009-0130-8 . hal-00380703

\section{HAL Id: hal-00380703 https://hal.science/hal-00380703}

Submitted on 4 May 2009

HAL is a multi-disciplinary open access archive for the deposit and dissemination of scientific research documents, whether they are published or not. The documents may come from teaching and research institutions in France or abroad, or from public or private research centers.
L'archive ouverte pluridisciplinaire HAL, est destinée au dépôt et à la diffusion de documents scientifiques de niveau recherche, publiés ou non, émanant des établissements d'enseignement et de recherche français ou étrangers, des laboratoires publics ou privés. 


\section{Marc Honnorat · Jérôme Monnier · Nicolas Rivière · Étienne Huot · François-Xavier Le Dimet \\ Identification of equivalent topography in an open channel flow using Lagrangian data assimilation}

Received: date / Accepted: date

\begin{abstract}
We present a Lagrangian data assimilation experiment in an open channel flow above a broad-crested weir. The observations consist of trajectories of particles transported by the flow and extracted from a video film, in addition to classical water level measurements. However, the presence of vertical recirculations on both sides of the weir actually conducts to the identification of an equivalent topography corresponding to the lower limit of a surface jet. In addition, results on the identification of the Manning coefficient may allow to detect the presence of bottom reciruclations.
\end{abstract}

\section{Introduction}

The numerical simulation of hydraulic systems require a precise modeling of the physics involved in the considered flows. Hydraulic models are built on physical assumptions and require information on the value of parameters, initial and boundary conditions in order to forecast a flow state which corresponds as well as possible to reality. To this purpose, data assimilation methods make it possible to combine optimally mathematical information contained in the equations of the model and physical information from observations. Such methods have been used in river models for the identification of model parameters in one dimensional channels [11,17,27] as well as for bidimensional shallow water equations [2, 3, 7, 12, 15, 19, 22, 23]. Let us point out that one

M. Honnorat $\cdot$ J. Monnier

INPG, INRIA, Lab. Jean Kuntzmann (LJK)

B.P. 53, 38041 Grenoble Cedex 9, France.

E-mail: marc.honnorat@imag.fr

N. Rivière

LMFA, INSA Lyon, France.

É. Huot

INRIA Rocquencourt, France.

F.-X. Le Dimet

UJF, INRIA, Lab. Jean Kuntzmann (LJK)

B.P. 53, 38041 Grenoble Cedex 9, France. of the main difficulties is to match real observations using a mathematical model that is naturally incomplete.

Commonly available observation data consist of water level measurements at gauging stations but remain very sparse in space. Recently, remote sensing techniques have been explored with the hope of providing new kinds of observations on hydraulic systems. In particular, image velocimetry techniques have been investigated [6,24] in order to reconstruct a dense field of surface velocity.

Observation of successive positions of passive particles transported on the surface of water can bring valuable information on the dynamics of the flow. In oceanography, data assimilation of drifting buoys positions has been experimentally used to improve numerical ocean models, either using Kalman filter [4, 18, 28] or variational based methods (see [16] for a shallow water model and more recently [26] for a primitive equations circulation model of the ocean).

Concerning river hydraulics, variational data assimilation of Lagrangian observations in a river model based on bidimensional shallow water equations was introduced in [14]. Numerical experiments using synthetic observations show that in addition to traditional water depth measurements, the use of particle trajectories can improve significantly the identification of model parameters.

In the current paper, we extend the methodology developed in [14] to a real application. We present experimental results of Lagrangian data assimilation in an open channel for the identification of a local topography among other parameters. The Lagrangian data consist of trajectories of paper confetti spread on the surface and observed by a video camera. The objective of the experiment is to catch the shape of a broad-crested weir positioned widthwise in the channel. However, the presence of vertical recirculations on both sides of the weir invalidates the shallow water model. Instead, the present configuration leads to the identification of an equivalent topography corresponding to the lower limit of a surface jet. Additional results suggest that the value of the Manning coefficient may allow to detect the presence of these bottom recirculations in the identified topography. Initial conditions corresponding to a permanent flow are identified as well. 
The experimental setup is described in Section 2 and the method for the extraction of observations from images is detailed in Section 3. In Section 4, we introduce the equations used for the modeling of the flow and the transport of particles. Section 5 is devoted to the description of the Lagrangian data assimilation method, following [14]. Finally, numerical results of data assimilation are presented and commented in Section 6 .

\section{Experimental setup}

The measurements are conducted in a channel with a rectangular cross section. The width is $L=0.805 \mathrm{~m}$ and the length of the observation area in the longitudinal direction is $1.1 \mathrm{~m}$ (see Fig. 1 for a schematic view). A broad-crested weir, made of a wooden plank, is placed on the bottom widthwise in abscissa $x_{1}=0.43 \mathrm{~m}$. This weir has a rectangular cross section, of $94 \mathrm{~mm}$ long and $37 \mathrm{~mm}$ high. The channel slope is $0.18 \%$.

The water enters the channel through honeycomb and wire netting buffers. A constant discharge of $\bar{q}=20 \mathrm{l} / \mathrm{s}$ is imposed at the inflow and the free surface is set downstream to the level $\bar{z}_{s}=0.107 \mathrm{~m}$, thanks to an adjustable tailgate. The corresponding Reynolds number is $R e=75190$. As the channel walls are made of PVC, the flow is hydraulically smooth with this value of the Reynolds number and the corresponding Manning roughness coefficient is therefore estimated to $n=0.0105 \mathrm{~s} / \mathrm{m}^{\frac{1}{3}}$. The boundary conditions are maintained until a mean permanent flow is reached. The observations were carried out in this hydraulic configuration.

Observations. The discharge is measured by an electromagnetic flow meter with an error of $\pm 0.05 \mathrm{l} / \mathrm{s}$. The water levels are measured thanks to a moveable point gauge, with an error of $\pm 0.15 \mathrm{~mm}$. Figure 1 shows the positions of water level measurements with black dots between the abscissa $x=0.18 m$ and $x=0.85 m$ in the local coordinate system of the observation area.

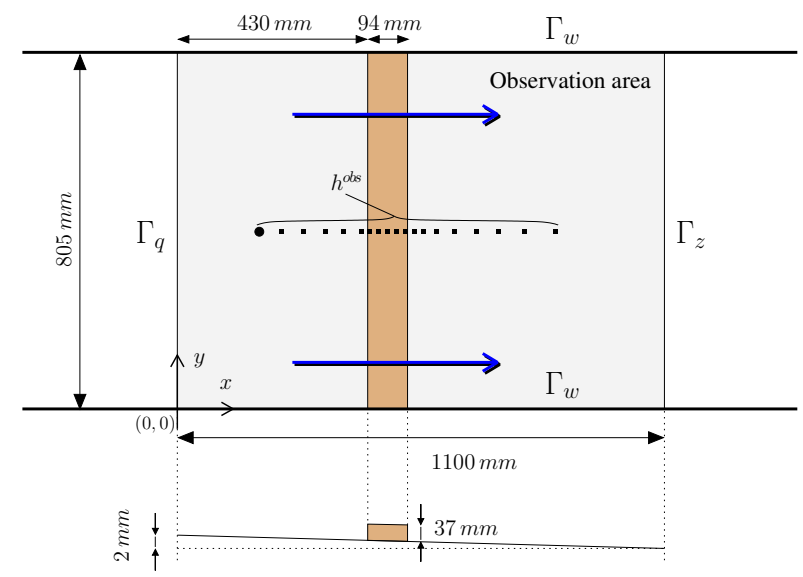

Fig. 1 Schematic description of the experimental setup.

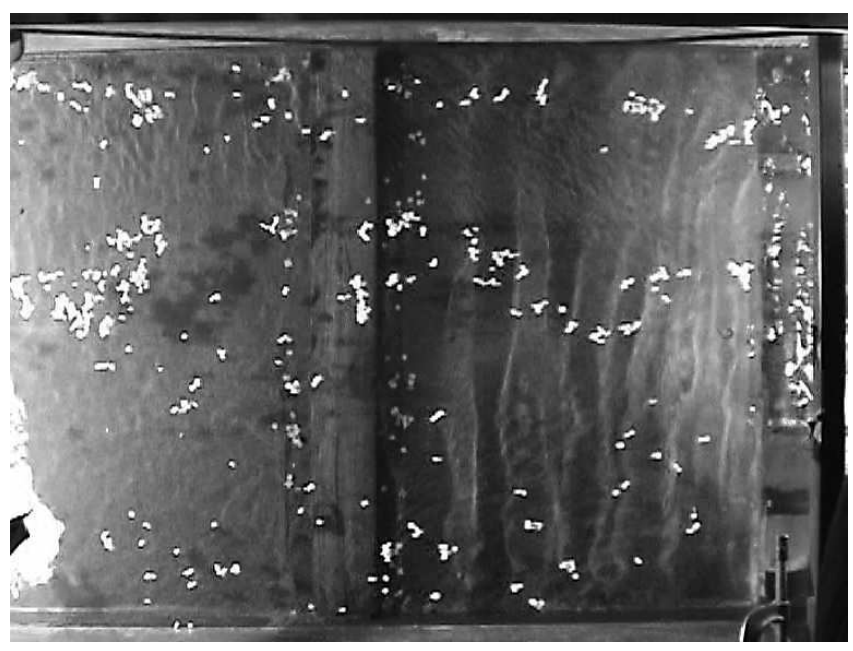

Fig. 2 Image extracted from a video sequence used for the experiment. Paper confetti transported on the water surface (from left to right).

Two kinds of observations coming from the experimental configuration are taken into account for the data assimilation experiment:

- a single surface elevation measurement, located at the abscissa $x=0.18 \mathrm{~m}$ and denoted by the bold circle in Fig. 1,

- a video sequence of paper bits drifted by the water. A video camera has been placed vertically above the obstacle to record the positions along time of confetti dropped on the surface upstream from the observation area.

All the observations that are not involved in data assimilation will be used for the validation of numerical results.

Parameters to be identified. The aim of the data assimilation experiment, presented in Section 5, is to identify the shape of the weir in the channel starting from the initial assumption of a flat bottom and using a shallow water model. In addition, we look for compatible values for the Manning coefficient as well as the initial conditions of the flow. A correlation coefficient between the surface velocity and the depth-averaged shallow water velocity will be also identified.

\section{Data processing}

An image extracted from a video sequence used in the data assimilation experiment is shown in Fig. 2 As we can see, several paper bits are often clustered together. Each cluster can easily be identified and tracked along the video sequence using a correlation-based matching method. A background image has been recorded without any confetti. In order to be less dependant of illumination conditions, each image is pre-processed by subtracting the background. The tracking of confetti clusters is then done as follows: 
1. For each cluster coming into the camera field, a $M \times M$ pixel square is initialized on the center of the cluster. Let $p=(u, v)$ denote the coordinates of the center of this square in frame \#i.

2. In the next frame $\# i+1$, we then look for the pixel with coordinates $\mathbf{p}^{\prime}=\left(u^{\prime}, v^{\prime}\right)$ that minimizes the correlation:

$$
C\left(\mathbf{p}, \mathbf{p}^{\prime}\right)=\sum_{x=-M}^{M} \sum_{y=-M}^{M}\left[I(u-x, v-y)-I^{\prime}\left(u^{\prime}-x, v^{\prime}-y\right)\right]^{2},
$$

where $I$ and $I^{\prime}$ correspond to the luminance of pixels respectively in frame $\# i$ and $\# i+1$.

It should be noted that there exist a lot of correlation measures proposed in computer vision literature; see for example [5]. We have chosen here one of the simplest.

By recording the coordinates $\mathbf{p}$ frame after frame, we obtain a set of coordinates corresponding to the trajectory of a cluster along the sequence. Tracking several clusters, we finally obtain a set of $N_{p}=256$ trajectories. These raw trajectories were then filtered by a reconstruction process based on a local averaging in time and space, as described in details in [14]. In Fig. 3(a), the longitudinal coordinate $x$ of a

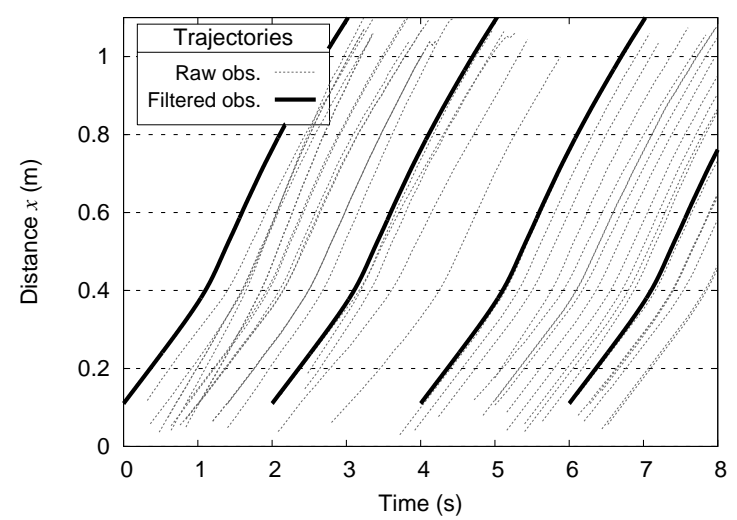

(a) Axes $t-x$

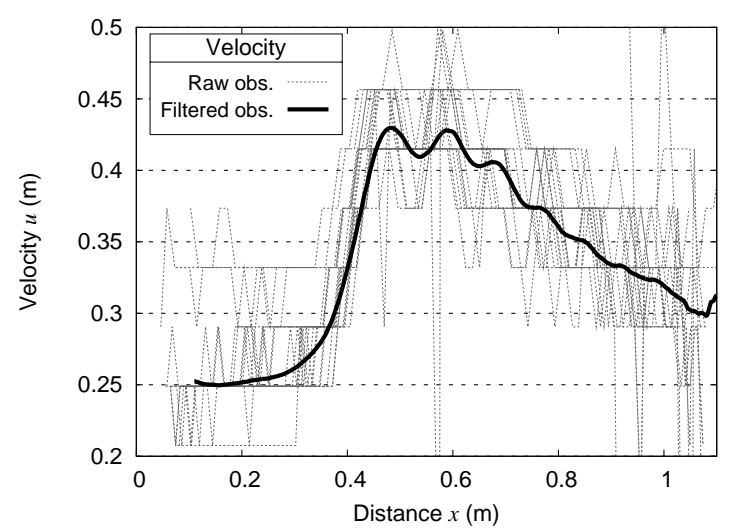

(b) Axes $x-u$

Fig. 3 Trajectories and longitudinal velocity of the observed particles. Thin line : raw observations from video images. Bold line : filtered trajectories. series of trajectories is plotted against the time $t$ in seconds. In Fig. 3(b), the longitudinal velocity is plotted against the first space variable. One can notice a quantization of the velocity of the raw observations. This phenomenon is due to the sampling of the video image: from one image to the following, the shifting of a particle is whole number of pixels and its velocity is therefore a discrete value. However, this quantization is strongly smoothed by the filtering process.

\section{Forward model}

In order to carry out numerical simulations of this flow, we use a river hydraulics model based on the bidimensional shallow water equations in a conservative formulation.

Shallow Water equations. The state variables are the water depth $h$ and the local discharge $\mathbf{q}=h \mathbf{u}$, where $\mathbf{u}$ is the depth-averaged velocity vector:

$$
\left\{\begin{array}{l}
\partial_{t} h+\operatorname{div}(\mathbf{q})=0 \\
\partial_{t} \mathbf{q}+\operatorname{div}\left(\frac{1}{h} \mathbf{q} \otimes \mathbf{q}\right)+\frac{1}{2} g \nabla h^{2}+g h \nabla z_{b}+g \frac{n^{2}\|\mathbf{q}\|}{h^{7 / 3}} \mathbf{q}=0 \\
\underline{I . C .} \quad h(0)=h^{0}, \mathbf{q}(0)=\mathbf{q}^{0} .
\end{array}\right.
$$

where $g$ is the magnitude of the gravity, $z_{b}$ is the bed elevation, $n$ is the Manning roughness coefficient, $h^{0}$ and $\mathbf{q}^{0}$ are the initial conditions for the state variables.

Boundary conditions. Concerning boundary conditions, a scalar discharge $\bar{q}$ is prescribed at the inflow $\Gamma_{q}$, a water elevation $\bar{z}_{s}$ at the outflow $\Gamma_{z}$ and the Riemann invariant is constant along the outgoing characteristic. For the lateral walls $\Gamma_{w}$, a slip condition on the velocity is prescribed:

$\underline{\text { B.C. }}\left\{\begin{array}{l}\left.(\mathbf{q} \cdot \mathbf{n})\right|_{\Gamma_{q}}=-\bar{q},\left.(\mathbf{q} \cdot \mathbf{n})\right|_{\Gamma_{w}}=0, \\ \left.\left(\partial_{\mathbf{n}} h\right)\right|_{\Gamma_{q} \cup \Gamma_{w}}=0, \\ h_{\Gamma_{z}}=\bar{z}_{S}-\left.z_{b}\right|_{\Gamma_{z}},\left.\partial_{\mathbf{n}}(\mathbf{u} \cdot \mathbf{n}+2 c)\right|_{\Gamma_{z}}=0,\end{array}\right.$

where $c=\sqrt{g h}$ denotes the local wave celerity. Boundary conditions on $\Gamma_{q}$ and $\Gamma_{z}$ are only valid for sub-critical flows, i.e. when $\|\mathbf{u}\|<c$. The model state variables $(h, \mathbf{q})$ are completely determined by the value of model parameters, initial conditions and boundary conditions gathered in the control vector $\mathbf{k}=\left(h^{0}, \mathbf{q}^{0}, n, z_{b}, \bar{q}, \bar{z}_{s}\right)$.

Transport model. Let us consider a set of $N$ particles transported by the flow. We state that their trajectories $X_{i}(t)$ are solutions of the following ODEs:

$$
\begin{aligned}
& \text { For } i=1, \ldots, N: \\
& \left\{\begin{array}{l}
\frac{d}{d t} X_{i}(t)=\mathbf{v}\left(X_{i}(t), t\right) \\
X_{i}\left(t_{i}^{0}\right)=x_{i}^{0}
\end{array} \quad \forall t \in\right] t_{i}^{0}, t_{i}^{f}[
\end{aligned}
$$

where $\mathbf{v}$ is the transport velocity of the particles, $t_{i}^{0}$ and $t_{i}^{f}$ are the time when the particle enters and leaves the observation domain. In the following, the transport velocity is related to 
the shallow water model velocity by a multiplicative constant: $\mathbf{v}=\gamma \mathbf{u}$. Here, $\gamma$ will be an additional control variable. The free surface velocity is usually greater than the depthaveraged velocity. Graf [10] indicates that for uniform fullydeveloped flows, $\gamma$ ranges from 1.1 to 1.25. Moreover, an acceleration of the flow correspond to a decrease of $\gamma$ while it increases in a decelerating flow.

This set of ODEs is weakly coupled with the shallow water model since the state variable of the latter is not dependent on the solution of the former.

Implementation. The shallow water model (2)-(3) is implemented using a finite volume scheme in the software Dassflow ${ }^{1}$ [13], designed for numerical simulation of river hydraulics, especially for parameter identification and variational data assimilation.

\section{Lagrangian data assimilation}

Variational data assimilation [20,29] is based on optimal control theory [21] and consists in identifying the control vector $\mathbf{k}$ that minimizes a cost function measuring the discrepancy between the state variable of the model and data obtained from the observation of the physical system.

Lagrangian data assimilation consists in using observations described by Lagrangian coordinates in a data assimilation process. Here, we consider observations of passive particles transported on the surface of the flow. However, the state of the flow is described in Eulerian coordinates by the shallow water model. Following [14], the link between Lagrangian data and the classical Eulerian variables of the shallow water model is made by the transport model.

Observations. We consider two kinds of observations. The first one consists in classical, Eulerian observations of the water depth in some locations of the physical domain, denoted by $h^{o b s}(t)$. The second one consists in trajectories of physical particles transported by the water flow. These Lagrangian observations are denoted by $X_{i}^{o b s}(t)$.

Cost function. In order to take into account both kinds of observations, we build a composite cost function measuring the discrepancy between observation data and model state variables:

$$
\begin{aligned}
j(\mathbf{k}) & =\frac{1}{2} \int_{0}^{T}\left\|C h(t)-h^{o b s}(t)\right\|_{o b s}^{2} d t \\
& +\frac{\alpha_{t}}{2} \sum_{i=1}^{N} \int_{t_{i}^{0}}^{t_{i}^{f}}\left|X_{i}(t)-X_{i}^{o b s}(t)\right|^{2} d t+\frac{\alpha_{p}}{2}\|D \mathbf{k}\|_{k}^{2},
\end{aligned}
$$

where $\mathbf{k}=\left(h^{0}, \mathbf{q}^{0}, n, z_{b}, \gamma\right)$ denotes the control vector and the constants $\alpha_{t}$ and $\alpha_{p}$ are scaling parameters. The operator $C$ computes the restriction of the model state variable $h$ to the space of Eulerian observations on which is defined the norm $\|\cdot\|_{o b s} . D$ is a differential operator and $\|\cdot\|_{k}$ is a norm defined

\footnotetext{
1 <http://dassflow.gforge.inria.fr/>
}

on the space of controls. The first term measures the discrepancy between water depth observations and model state variable. The second term measures the distance between virtual particles of the transport model and observations of trajectories, while the third one is a regularization term.

Optimization problem. We seek to minimize the cost function, ie. to identify the control vector $\mathbf{k}^{o p t}$ that is solution to the following optimization problem:

$\mathbf{k}^{\text {opt }}=\min _{\mathbf{k}} j(\mathbf{k})$.

A quasi-Newton method can be used to that purpose, which requires the computation of the gradient of the cost function. Here, we use the M1QN3 algorithm [9] based on the BFGS formula.

Adjoint model The adjoint method [21] makes it possible to compute efficiently all partial derivatives of the cost function $j$ with respect to the components of the control vector $\mathbf{k}$. The derivatives can be expressed as simple functions of the solutions to an adjoint model. In the case of the system (2)(3)-(4), the latter is weakly coupled. A backward integration in time of an adjoint transport model followed by a backward integration in time of an adjoint shallow water model are sufficient to compute all components of $\nabla j(\mathbf{k})$. This method has been implemented in the software Dassflow. A complete description is given in [14,12].

\section{Identification results}

In this section, we present a data assimilation experiment in order to identify model control variables. The objective is to catch the shape of the broad-crested weir positioned widthwise in the channel with the shallow water model, knowing that the latter is incomplete in regard to the considered flow.

Reference simulation. Beforehand, a direct simulation, thereafter called reference shallow water simulation, is carried out using all available informations about the hydraulic configuration - boundary conditions, topography of the channel, estimated Manning coefficient. The Fig. 4](a) shows a comparison between the simulated water depth (dashed line) and the observations $h^{\text {obs }}$ (black dots). One can notice a good agreement between the two curves, which shows that the model is able to represent correctly water depth observations. The Fig. 4 (b) shows the same comparison for longitudinal velocities. Here, the black dots represent the discharge velocity computed from water depth observations: $u^{o b s}=\frac{\bar{q}}{L \times h^{o b s}}$, where $L$ is the channel width. In addition, the continuous line corresponds to the longitudinal velocity of a filtered trajectory. A significant discrepancy can be noticed between this surface velocity and the discharge velocity. In the first part of the flow (for $x<0.5 \mathrm{~m}$ ), this gap could be modeled by a multiplicative constant $\gamma$ as in equation (4) with a value roughly approximated to 1.1 .

In the second part of the flow, however, this relation does not stand up anymore. Indeed, the weir introduces a sharp 


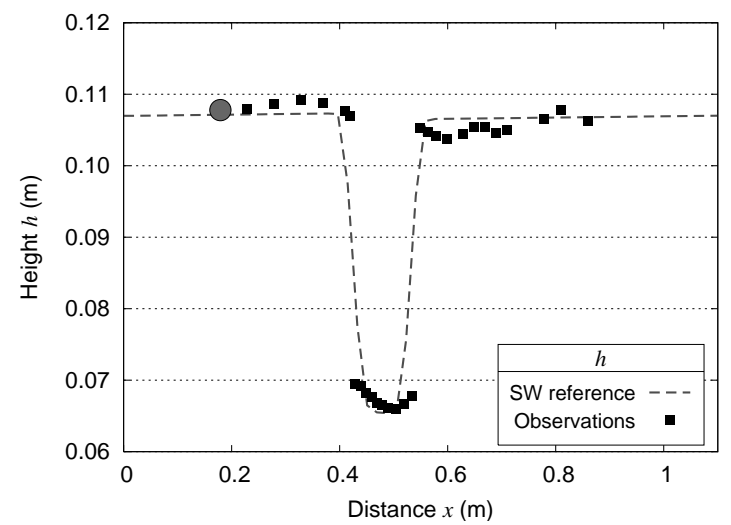

(a) Water depth

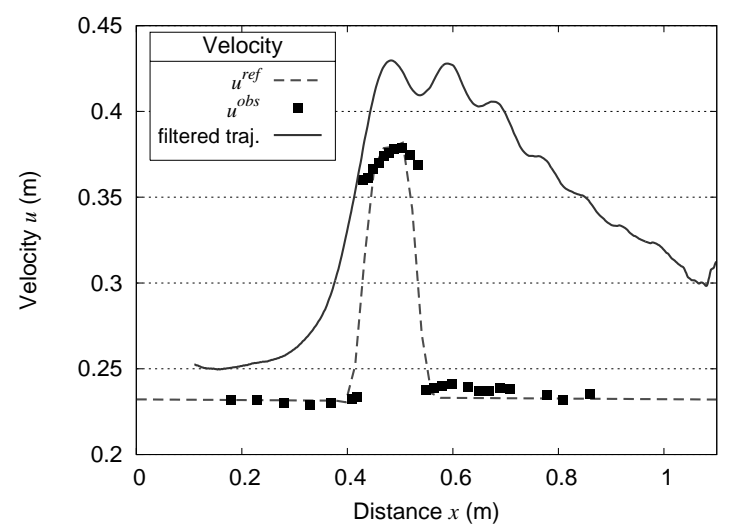

(b) Longitudinal velocity

Fig. 4 Comparison between the reference simulation and observations (a) Water depth and (b) longitudinal velocity. Dashed line: shallow water reference simulation. Black squares: data computed from water depth measurements. Bold circle: measurement used for data assimilation. Continuous line: velocity of a filtered particle.

topography gradient. Such an obstacle in the flow typically produces a surface jet and a bottom recirculation [8,30], as shown schematically in Fig. 5 These recirculations comprise vertical accelerations, which are not compatible with the shallow water equations. A full free-surface 3D NavierStokes model would be more suitable to simulate these structures. One can nevertheless carry out data assimilation using the water depth measurements and the observations of trajectories in order to determine the optimal set of parameter that combine optimally this data with the present shallow water model.

Data assimilation experiment. Given the observation of the water depth at the single point $x_{o b s}=0.18 \mathrm{~m}$, highlighted in Fig. 4 (a), in addition to $N_{m}=150$ filtered trajectories denoted $X_{j}^{\text {obs }}(t)$, we seek to identify the topography $z_{b}$, the Manning coefficient $n$, the initial conditions $h^{0}$ and $\mathbf{q}^{0}$ as well as the coefficient $\gamma$ from the transport model (4). Initial assumptions on the value of these parameters are adopted: a flat bottom without obstacle, Manning coefficient uniformly set to $n=0.01, \gamma$ coefficient uniformly set to 1.1 . Moreover, the first guess value for the initial conditions correspond to the steady state of the flow obtained with the other parameters.

The data assimilation experiment consists in minimizing the following cost function:

$$
\begin{aligned}
j\left(z_{b}, n, h^{0}, \mathbf{q}^{0}, \gamma\right) & =\frac{1}{2} \int_{0}^{T} \int_{0}^{L}\left|h\left(x_{o b s}, y ; t\right)-h^{o b s}(t)\right|^{2} d y d t \\
+ & \frac{\alpha_{t}}{2} \sum_{j=1}^{N_{m}} \int_{t_{j}^{0}}^{t_{j}^{f}}\left|X_{j}(t)-X_{j}^{o b s}(t)\right|^{2} d t \\
+ & \frac{\alpha_{z}}{2}\left\|\Delta z_{b}\right\|^{2}+\frac{\alpha_{h}}{2}\left\|\Delta h^{0}\right\|^{2}+\frac{\alpha_{q}}{2}\left\|\Delta \mathbf{q}^{0}\right\|^{2},
\end{aligned}
$$

where the scaling coefficient $\alpha_{t}$ is set to $10^{-5}$ in order to respect an initial balance between the first two terms. The weights $\alpha_{z}, \alpha_{h}$ and $\alpha_{q}$ of the regularization terms are set respectively to $10^{-9}, 10^{-12}$ and $10^{-12}$.

Identification results. The results of the identification are shown in Fig. 6. For each control variable, the identified value is drawn with a continuous line and the reference value from the reference shallow water simulation is drawn with a dotted line for comparison. In addition, the evolution of the cost function and the norm of its gradient is plotted against the number of iterations of the minimization process in Fig. 6 (f). The value of the cost function was divided by 10000 to reach $5 \times 10^{-6}$, which indicates a good performance of the minimization. This is confirmed by the similarity between velocity of the observed particles and the model surface velocity $\gamma \mathbf{u}$ as shown in Fig. 7 .

Concerning the identified control variables, Fig. 6 (a) shows that the identified topography does not reproduce the shape of the obstacle: instead of a sharp rectangular profile, one gets a bump whose dispersion in the longitudinal direction is much more important. However, its amplitude is acceptable.

A physical interpretation to this result can be provided. Indeed, the extension of the identified topography on both sides of the real obstacle reveal bottom recirculations upstream and downstream of the weir, as shown in Fig. 5. In any point of the domain, the discharge in the shallow water equations is equal to the integration of that of the threedimensional flow. Therefore, the integrated discharge over a vertical vortex is zero. The shallow water model cannot reproduce such a structure, but only the upper part of the flow taking part in the effective discharge, ie. the region between the water surface and the line of zero discharge, denoted parting line in Fig. 5. The latter is the center line of the mixing layer between the bottom recirculation and the surface jet.

From a quantitative point of view, it is possible to validate this hypothesis by looking at the ratio between the length $L_{r}$ of the downstream recirculation in the longitudinal direction and the height $w$ of the weir, as shown in Fig. 5. Given the identified topography in Fig. 6 (a) and assuming that it actually covers the real obstacle and bottom recirculations, this ratio $\frac{L_{r}}{w}$ is approximately 12 .

For a backward facing step, Adams and Johnston [1] show that this ratio depends on the downstream and up- 


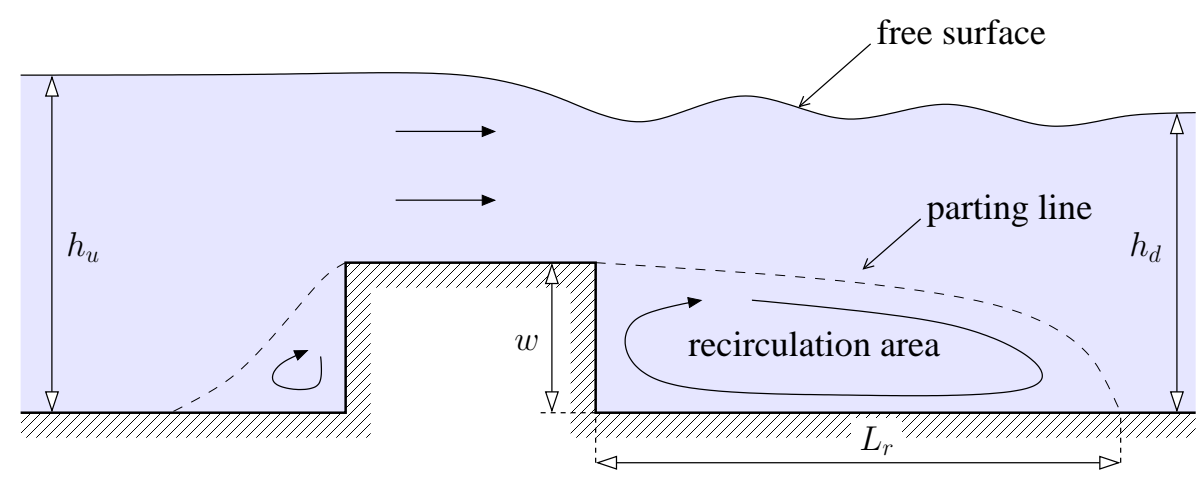

Fig. 5 Schematic view of upstream and downstream recirculations around a rectangular immersed obstacle.

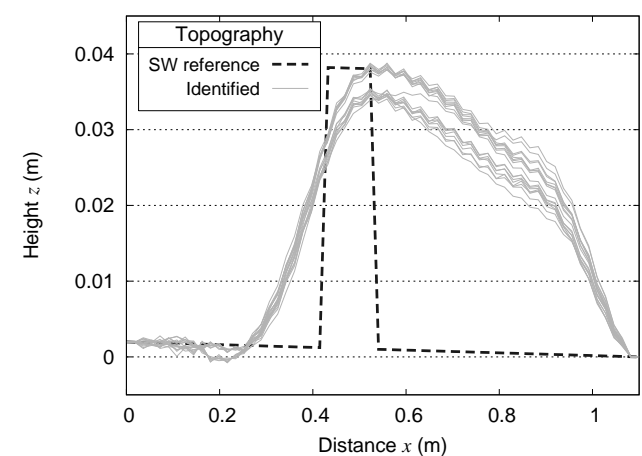

(a) Topography $z_{b}$.

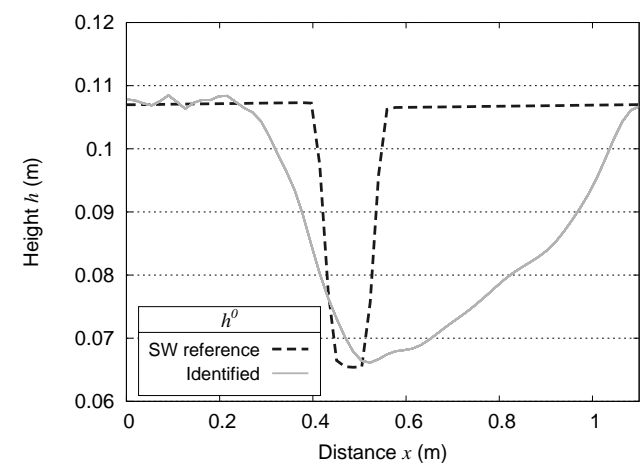

(c) Initial water depth $h^{0}$.

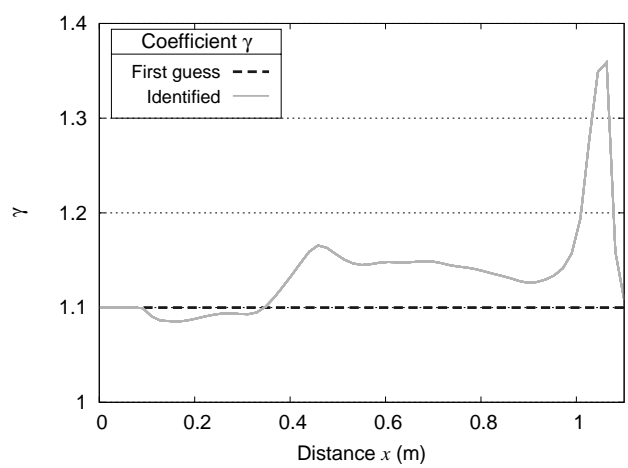

(e) $\gamma$ coefficient.

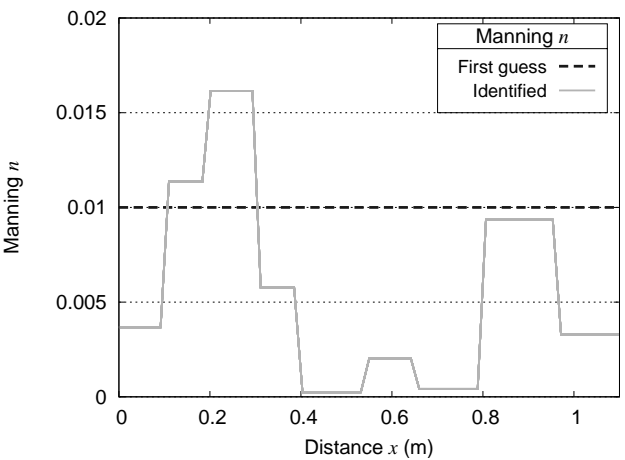

(b) Manning coefficient.

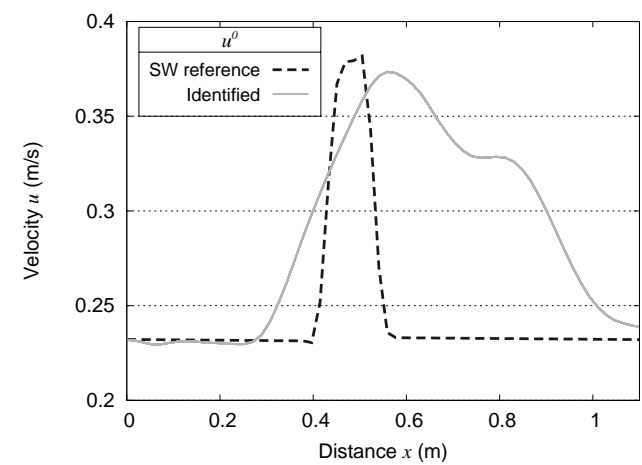

(d) Initial longitudinal velocity $u^{0}$.

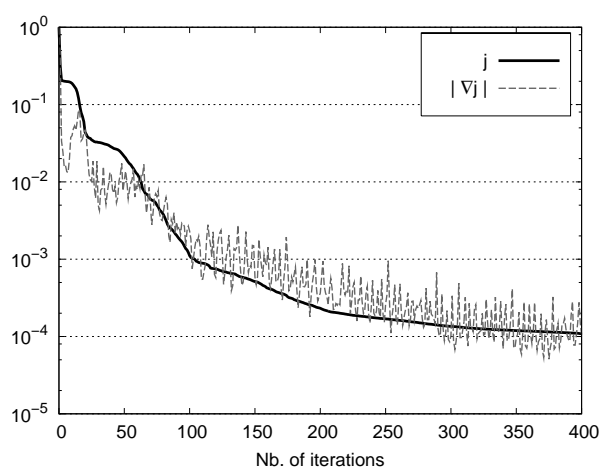

(f) Cost function.

Fig. 6 Identification of the topography, the Manning coefficient, the initial conditions and the $\gamma$ coefficient from water depth measurements and observations of particle trajectories. Dashed line: reference parameters. Continuous line: identified values. 


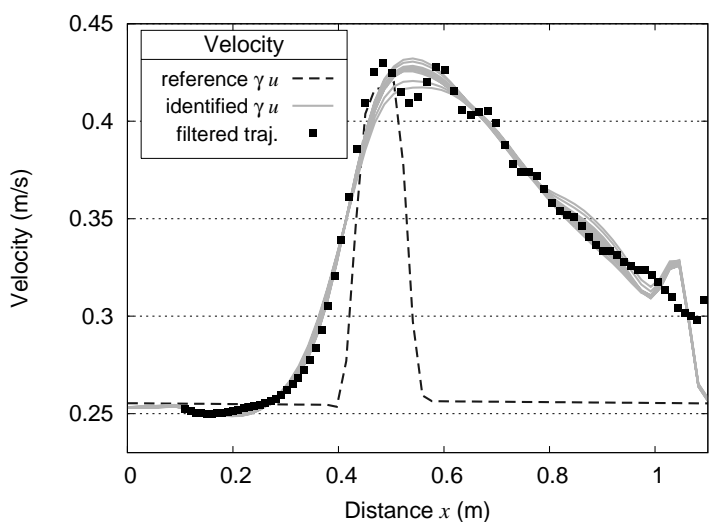

Fig. 7 Comparison between velocity of filtered particles and model surface velocity. Thin line: longitudinal model surface velocity $\gamma u$. Black squares: longitudinal velocity corresponding to a filtered trajectory used for data assimilation.

stream depths but also on the Reynolds number ; the influence of the latter disappears for values higher than $10^{4}$, condition fulfilled in the present work as well as in the papers cited below. In a case closer to our problem, Fritz and Hager [8] have studied experimentally flow characteristics downstream of submerged symmetric trapezoidal weirs. In the case of a surface jet, they established the following relation:

$\frac{L_{r}}{w}=6.8 \frac{h_{d}}{w}\left(1-\frac{h_{d}-w}{h_{u}-w}\right)^{\frac{1}{6}}$,

where $h_{u}$ and $h_{d}$ are respectively the upstream and downstream water levels. Using our data $(w=37 \mathrm{~mm}$, $h_{u}=109 \mathrm{~mm}$ and $h_{d}=107 \mathrm{~mm}$ ), this relation gives a ratio $\frac{L_{r}}{w}=10.8$.

In our case, however, the obstacle is rectangular shaped, not trapezoidal. Nakayama and Yokojima [25] carried out numerical simulations based on free surface Navier-Stokes equations in a configuration similar to ours. Their results show a ratio $\frac{L_{r}}{w}$ close to 11 . This indicates that the order of magnitude of the ratio given by equation (8) is still valid for a rectangular shaped obstacle. This physical likelihood tends to confirm our hypothesis. So, instead of the bottom geometry, the numerical model reproduces the line of zero discharge, i.e. the upstream recirculation, the weir crest and the downstream recirculation.

The physical behavior of the flow being stated, the values of the other control variables must be analyzed. Concerning the initial water depth and longitudinal velocity, shown in Fig. 6 (c) and (d), they correspond rather well to a steady state. As expected, the $\gamma$ coefficient, whose evolution is depicted in Fig. 6(e), is slightly lower than its reference value in the converging - and therefore accelerating - flow upstream the weir. Conversely, it is above its reference value in the diverging - and therefore decelerating - flow over the downstream recirculation. The sudden increase in the last part of the observation window may be related to the mismatch between the velocity of the particles and the transport velocity $\gamma \mathbf{u}$ in the same region, as can be observed in Fig. 7 . This may be explained by a very small sensitivity of the control variables to the observations in this area.

Finally, it is interesting to comment the evolution of the identified Manning roughness coefficient (Figure 6 (b)). Downstream and upstream of the weir and the recirculations, its value is close to the experimental one (from 0.009 to 0.016 instead of 0.01 ), except at the boundaries of the observation window. One can notice a strong decrease of the coefficient in the region corresponding to the identified "equivalent weir". It stands for the huge modifications of the vertical velocity profiles in this region, compared to the uniform flow velocity profiles required by the use of a Manning roughness coefficient: shear stresses smaller in the two mixing layers than on the bottom shear layer and uniform velocity profiles on the weir crest. Although this should be confirmed on a wider range of experiments, such values of $n$ close to zero may allow to detect the presence of bottom recirculations in the identified topography.

\section{Conclusion}

In this paper, we have presented a lagrangian data assimilation experiment in an open channel flow. The channel had a rectangular cross section and was fitted with a broad-crested weir. With a mean permanent flow, vertical recirculations were present upstream and downstream from the weir. Small paper bits were spread on the surface of water and their trajectories were recorded with a video camera placed vertically above the weir.

We developed a numerical model of this flow configuration based on the shallow water equations, combined with a transport model for the particles. By means of a variational data assimilation, we sought to identify the topography of the channel, using the observations of trajectories in addition to traditional water level measurements at one single point.

The first striking result of this experiment is that the identified topography does not correspond to the real bottom geometry of the channel, but to the line of zero discharge covering the upstream and downstream recirculations in addition to the weir. Indeed, vertical recirculations are threedimensional fluid structures, and cannot be plainly represented by a shallow water model.

Secondly, the results concerning the Manning coefficient suggest that the identification of values of $n$ that are close to zero may be used as a criterion to distinguish a real topography from an apparent topography and therefore detect the presence of recirculations.

Finally, the identified initial conditions correspond closely to a steady state, which is in accordance with the nature of the real flow. These first results seem promising since the model reproduces correctly the topography and water depth in a case of complex flow, i.e. involving flow separations. Nevertheless, future work should be carried out to confirm these results through an evaluation of the model 
with other experiments, either in absence of flow separation or with variations of the channel width inducing transverse velocities.

Acknowledgements The authors would like to thank the French Region Rhône-Alpes for supporting this work in the framework of the "Thématique prioritaire Développement durable (Risques Naturels)".

\section{References}

1. Adams, E.W., Johnston, J.P.: Effects of the separating shear layer on the reattachment flow structure. Part 2: reattachment lenght and wall shear stress. Experiments In Fluids 6, 493-499 (1988)

2. Atanov, G.A., Evseeva, E.G., Meselhe, E.A.: Estimation of roughness profile in trapezoidal open channels. Journal of Hydraulic Engineering 125(3), 309-312 (1999)

3. Bélanger, E., Vincent, A.: Data assimilation (4D-VAR) to forecast flood in shallow-waters with sediment erosion. Journal of Hydrology 300(1-4), 114-125 (2005)

4. Carter, E.F.: Assimilation of lagrangian data into a numerical model. Dynamics of Atmospheres and Oceans 13(3-4), 335-348 (1989)

5. Chambon, S., Crouzil, A.: Towards correlation-based matching algorithms that are robust near occlusions. In: International Conference on Pattern Recognition - ICPR 2004, pp. 20-23. Cambridge, United-Kingdom (2004)

6. Creutin, J.D., Muste, M., Bradley, A.A., Kim, S.C., Kruger, A.: River gauging using PIV techniques: a proof of concept experiment on the Iowa River. Journal of Hydrology 277, 182-194 (2003)

7. Ding, Y., Jia, Y., Wang, S.S.Y.: Identification of Manning's roughness coefficients in shallow water flows. Journal of Hydraulic Engineering 130(6), 501-510 (2004)

8. Fritz, H.M., Hager, W.H.: Hydraulics of embankment weirs. Journal of Hydraulic Engineering 124(9), 963-971 (1998)

9. Gilbert, J.C., Lemaréchal, C.: Some numerical experiments with variable storage Quasi-Newton algorithms. Mathematical Programming 45, 407-435 (1989)

10. Graf, W.H.: Hydraulique Fluviale. Presses Polytechniques et Universitaires Romandes, Lausanne, Switzerland (2000). In french

11. Hartnack, J., Madsen, H.: Data assimilation in river flow modelling. In: $4^{\text {th }}$ DHI software conference, $6-8$ june 2001. Scanticon Conference Center, Helsingør, Danemark (2001)

12. Honnorat, M.: Lagrangian data assimilation for river hydraulics simulations. Ph.D. thesis, INP Grenoble, LJK (2007). In French

13. Honnorat, M., Marin, J., Monnier, J., Lai, X.: DAssflow v1.0: a variational data assimilation software for $2 \mathrm{D}$ river flows. Research Report RR-6150, INRIA (2007)

14. Honnorat, M., Monnier, J., Le Dimet, F.X.: Lagrangian data assimilation for river hydraulics simulations. Computing and Visualization in Science, accepted (2007)

15. Hostache, R., Lai, X., Monnier, J., Puech, C., Paquier, A.: Assimilation of spatial distributed water levels into a shallow-water flood model. Part II: Moselle river. To appear (2007)

16. Kamachi, M., O'Brien, J.J.: Continuous data assimilation of drifting buoy trajectory into an equatorial Pacific Ocean model. Journal of Marine Systems 6, 159-178 (1995)

17. Khatibi, R.H., Williams, J.J.R., Wormleaton, P.R.: Identification problem of open-channel friction parameters. Journal of Hydraulic Engineering 123(12), 1078-1088 (1997)

18. Kuznetsov, L., Ide, K., Jones, C.K.R.T.: A method for assimilation of lagrangian data. Monthly Weather Review 131(10), 2247-2260 (2003)

19. Lai, X., Monnier, J.: Assimilation of spatial distributed water levels into a shallow-water flood model. Part I: method and toy test case. To appear (2007)
20. Le Dimet, F.X., Talagrand, O.: Variational algorithms for analysis and assimilation of meteorological observations: theoretical aspects. Tellus 38(A), 97-110 (1986)

21. Lions, J.L.: Optimal Control of Systems Governed by Partial Differential Equations. Springer-Verlag (1971)

22. Madsen, H., Hartnack, J., Sørensen, J.V.T.: Data assimilation in a flood modelling system using the Ensemble Kalman filter. In: CMWR-XVI. Copenhaguen, Danemark (2006)

23. Mazauric, C.: Data assimilation for hydraulic models. parameters estimation, sensitivity analysis and domain decomposition. Ph.D. thesis, Université Joseph Fourier, LMC-IMAG (2003). In french

24. Muste, M., Xiong, Z., Schöne, J., Li, Z.: Validation and extension of image velocimetry capabilities for flow diagnostic in hydraulic modeling. Journal of Hydraulic Engineering 130(3), 175185 (2004)

25. Nakayama, A., Yokojima, S.: Modeling free-surface fluctuation effects for calculation of turbulent open-channel flows. Environmental Fluid Mechanics 3, 1-21 (2003)

26. Nodet, M.: Variational assimilation of lagrangian data in oceanography. Inverse Problems 22, 245-263 (2006)

27. Roux, H., Dartus, D.: Parameter identification using optimization techniques in open-channel inverse problems. Journal of Hydraulic Research 43(3), 311-320 (2005)

28. Salman, H., Kuznetsov, L., Jones, C.K.R.T.: A method for assimilating lagrangian data into a shallow-water-equation ocean model. Monthly Weather Review 134(4), 1081-1101 (2006)

29. Talagrand, O., Courtier, P.: Variational assimilation of meteorological observations with the adjoint vorticity equation. I : Theory. Quarterly Journal of the Royal Meteorological Society 113(13111328) (1987)

30. Wu, S., Rajaratnam, N.: Submerged flow regimes of rectangular sharp-crested weirs. Journal of Hydraulic Engineering 122(7), 412-414 (1996) 